\title{
Statistics of passive tracers in three-dimensional magnetohydrodynamic turbulence
}

Angela Busse, Wolf-Christian Müller, Holger Homann, and Rainer Grauer

Citation: Phys. Plasmas 14, 122303 (2007); doi: 10.1063/1.2818770

View online: http://dx.doi.org/10.1063/1.2818770

View Table of Contents: http://pop.aip.org/resource/1/PHPAEN/v14/i12

Published by the American Institute of Physics.

\section{Related Articles}

A priori analysis of subgrid mass diffusion vectors in high pressure turbulent hydrogen/oxygen reacting shear layer flames

Phys. Fluids 24, 075114 (2012)

Hybrid simulations of metal particle nucleation: A priori and a posteriori analyses of the effects of unresolved scalar interactions on nanoparticle nucleation

Phys. Fluids 24, 075110 (2012)

Mitigation of preferential concentration of small inertial particles in stationary isotropic turbulence using electrical and gravitational body forces

Phys. Fluids 24, 073301 (2012)

Detection of turbulent thermal diffusion of particles in numerical simulations

Phys. Fluids 24, 075106 (2012)

Secondary energy growth and turbulence suppression in conducting channel flow with streamwise magnetic field Phys. Fluids 24, 074101 (2012)

\section{Additional information on Phys. Plasmas}

Journal Homepage: http://pop.aip.org/

Journal Information: http://pop.aip.org/about/about_the_journal

Top downloads: http://pop.aip.org/features/most_downloaded

Information for Authors: http://pop.aip.org/authors

\section{ADVERTISEMENT}

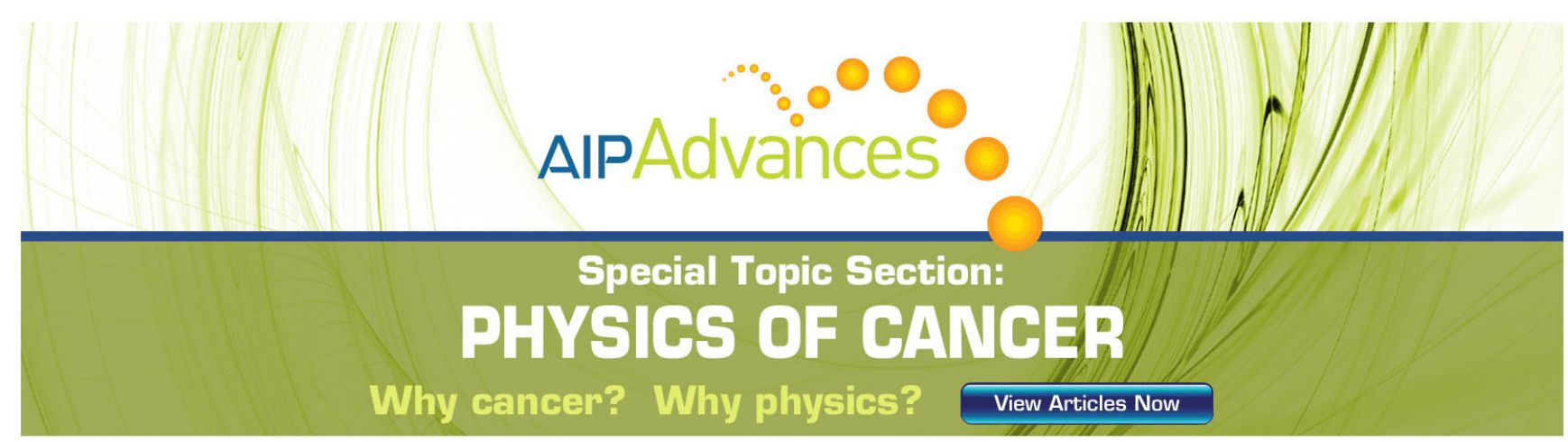




\title{
Statistics of passive tracers in three-dimensional magnetohydrodynamic turbulence
}

\author{
Angela Busse and Wolf-Christian Müller ${ }^{a)}$ \\ Max-Planck-Institut für Plasmaphysik, 85748 Garching, Germany \\ Holger Homann and Rainer Grauer \\ Theoretische Physik I, Ruhr-Universität Bochum, 44780 Bochum, Germany
}

(Received 20 June 2007; accepted 7 November 2007; published online 13 December 2007)

\begin{abstract}
Magnetohydrodynamic (MHD) turbulence is studied from the Lagrangian viewpoint by following fluid particle tracers in high resolution direct numerical simulations. Results regarding turbulent diffusion and dispersion as well as Lagrangian structure functions are presented. Whereas turbulent single-particle diffusion exhibits essentially the same behavior in Navier-Stokes and MHD turbulence, two-particle relative dispersion in the MHD case differs significantly from the Navier-Stokes behavior. This observation is linked to the local anisotropy of MHD turbulence which is clearly reflected by quantities measured in a Lagrangian frame of reference. In the MHD case the Lagrangian structure functions display a lower level of intermittency as compared to the Navier-Stokes case contrasting Eulerian results. This is not only true for short time increments [H. Homann, R. Grauer, A. Busse, and W.-C. Müller, J. Plasma Phys. 73, 821 (2007)] but also holds for increments up to the order of the integral time scale. The apparent discrepancy can be explained by the difference in the characteristic shapes of fluid particle trajectories in the vicinity of most singular dissipative structures. (C) 2007 American Institute of Physics.
\end{abstract}

[DOI: $10.1063 / 1.2818770]$

\section{INTRODUCTION}

During the last decade Lagrangian statistics of turbulent flows have been receiving increasing attention as new experimental techniques ${ }^{1-5}$ and high-resolution numerical simulations ${ }^{6-8}$ have enabled detailed studies of the dynamics of advected tracer particles.

The Lagrangian point of view is particularly suited for the study of the diffusive characteristics of turbulent flows. ${ }^{9,10}$ The diffusion and dispersion of tracer particles in neutral fluids has been the subject of various experimental (see, for example, Refs. 2, 11, and 12) and numerical studies (see, e.g., Refs. 6, 7, and 13). Related problems regarding the turbulent diffusion of magnetic fields and the influence of turbulent magnetic fields on particle diffusion have been investigated extensively in space and astrophysics, see, e.g., Refs. $14-19$, as well as with regard to magnetically confined nuclear-fusion plasmas (see, for example, Refs. 20-22). Moreover the investigation of Lagrangian velocity increments is a complementary approach that yields information about intermittency and the spatial structure of turbulence (see, e.g., Refs. 23 and 24).

In this paper a first effort is presented to identify differences of the Lagrangian statistics of turbulence in electrically conducting and electrically neutral media. To this end the results of several direct numerical simulations of threedimensional incompressible and macroscopically isotropic Navier-Stokes and MHD turbulence seeded with massless point particles (tracers) are analyzed.

The organization of the paper is as follows: In Sec. II a

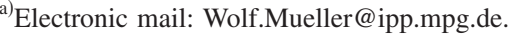

short overview of the numerical scheme used for tracking the tracer particles is given. In Sec. III the diffusion of single particles is discussed. In Sec. IV results regarding relative dispersion of particle pairs are presented. Section V deals with Lagrangian structure functions and their relation to the characteristic shapes of particle trajectories near structures of high dissipation. The conclusions are summarized in Sec. VI.

\section{NUMERICAL METHOD}

The incompressible MHD equations in dimensionless (Alfvénic) units are given by

$$
\begin{aligned}
& \partial_{t} \boldsymbol{\omega}=\nabla \times[\boldsymbol{v} \times \boldsymbol{\omega}-\boldsymbol{b} \times(\nabla \times \boldsymbol{b})]+\mu \Delta \boldsymbol{\omega}, \\
& \partial_{t} \boldsymbol{b}=\nabla \times(\boldsymbol{v} \times \boldsymbol{b})+\eta \Delta \boldsymbol{b}, \\
& \nabla \cdot \boldsymbol{v}=0, \\
& \nabla \cdot \boldsymbol{b}=0 .
\end{aligned}
$$

$\boldsymbol{v}$ is the velocity field, related to the vorticity by $\boldsymbol{\omega}=\nabla \times \boldsymbol{v}$, and $\boldsymbol{b}$ is the magnetic field. To obtain this form the MHD equations in Gaussian units (see, for example, Ref. 25) are normalized using a typical flow speed $V_{0}$, a length scale characteristic of its spatial variation $L_{0}$, and a characteristic magnetic field strength $B_{0}$. The interaction parameter, $B_{0}^{2}\left(4 \pi \rho_{0}\right)^{-1} / V_{0}^{2}$, in front of the Lorentz force term is set to unity by proper choice of $B_{0}$. Here $\rho_{0}=1$ is the uniform density of the fluid. The dimensionless kinematic viscosity $\mu$ and magnetic diffusivity $\eta$ are formally reciprocals of the kinetic and magnetic Reynolds number, respectively. In laboratory units, these dimensionless numbers are $\operatorname{Re}=L_{0} V_{0} / \nu^{*}$ 
TABLE I. Parameters of the numerical simulations: Taylor scale Reynolds number $R_{\lambda}=2 / 3\left[15 u_{\mathrm{rms}} E^{3 / 2} /(\varepsilon \mu)\right]^{1 / 2}$, root-mean-square velocity $u_{\mathrm{rms}}$, and magnetic field $b_{\text {rms }}$, kinetic energy dissipation rate $\varepsilon_{\text {kin }}$, magnetic energy dissipation rate $\varepsilon_{\text {mag }}, \varepsilon=\varepsilon_{\text {kin }}+\varepsilon_{\text {mag }}$, Kolmogorov length scale $\ell_{d}=\left(\mu^{3} / \varepsilon_{\text {kin }}\right)^{1 / 4}$, Kolmogorov time scale $\tau_{d}=\left(\mu / \varepsilon_{\text {kin }}\right)^{1 / 2}$, resolution $N^{3}$, number of tracer particles $N_{p}$. Run1 and Run3: Navier-Stokes simulations, Run2 and Run4: MHD simulations.

\begin{tabular}{|c|c|c|c|c|c|c|c|c|c|c|c|}
\hline$R_{\lambda}$ & $u_{\mathrm{rms}}$ & $b_{\text {rms }}$ & $\varepsilon_{\text {kin }}$ & $\varepsilon_{\mathrm{mag}}$ & $\mu=\eta$ & $d x$ & $\ell_{d}$ & $\tau_{d}$ & $N^{3}$ & $N_{p}$ & \\
\hline 190 & 0.82 & $\ldots$ & 0.23 & $\ldots$ & $8 \times 10^{-4}$ & $1.23 \times 10^{-2}$ & $6.9 \times 10^{-3}$ & $5.9 \times 10^{-2}$ & $512^{3}$ & $1.18 \times 10^{6}$ & Run1 \\
\hline 187 & 0.48 & 0.69 & 0.10 & 0.15 & $5 \times 10^{-4}$ & $1.23 \times 10^{-2}$ & $5.9 \times 10^{-3}$ & $7.1 \times 10^{-2}$ & $512^{3}$ & $1.18 \times 10^{6}$ & Run2 \\
\hline 316 & 0.18 & $\cdots$ & $3.5 \times 10^{-3}$ & $\ldots$ & $2 \times 10^{-4}$ & $6.14 \times 10^{-3}$ & $2.5 \times 10^{-3}$ & 0.12 & $1024^{3}$ & $5 \times 10^{6}$ & Run3 \\
\hline 185 & 0.22 & 0.32 & $1.0 \times 10^{-2}$ & $1.5 \times 10^{-2}$ & $3 \times 10^{-4}$ & $1.23 \times 10^{-3}$ & $7.1 \times 10^{-3}$ & 0.17 & $512^{3}$ & $5 \times 10^{5}$ & Run4 \\
\hline
\end{tabular}

and $R m=L_{0} V_{0} / \mu^{*}$, where $\nu^{*}$ and $\mu^{*}$ are kinematic viscosity and magnetic diffusivity given in dimensional units. The Navier-Stokes equations are obtained by setting $\boldsymbol{b}=0$ in Eqs. (1)-(4). The magnetic Prandtl number $\operatorname{Pr}_{m}=\mu / \eta$ is set to unity.

A number of direct numerical simulations of macroscopically isotropic turbulence are conducted by solving the incompressible MHD and Navier-Stokes equations by a standard pseudospectral method on a cubic grid with periodic boundary conditions. The pseudospectral treatment of the equations preserves the solenoidality of $\boldsymbol{\omega}$ and $\boldsymbol{b}$ down to machine accuracy. The solenoidality of the velocity field is ensured by its algebraic computation from the vorticity field in Fourier space. The computational grid has a resolution of $512^{3}$ (MHD, NS) and $1024^{3}$ (NS) grid points, respectively. Numerical parameters are summarized in Table I. While Run 1 and Run2 use a leapfrog scheme Run3 and Run4 use a Runge-Kutta scheme of third order. Aliasing errors are reduced by spherical mode truncation ${ }^{26}$ in Run1-Run3 and by a high-order Fourier smoothing ${ }^{27}$ in Run4. The standard small scale resolution requirement $k_{\max } \ell_{\mathrm{d}} \geq 1.5$ (Ref. 28) is approximately met in all simulations.

In this paper the Kolmogorov length and time scales are computed using the kinetic energy dissipation rate $\varepsilon_{\text {kin }}$ $=\mu \int_{V} d V \omega^{2}$ in both the Navier-Stokes and the MHD case,

$$
\ell_{d}=\left(\frac{\mu^{3}}{\varepsilon_{\mathrm{kin}}}\right)^{1 / 4}, \quad \tau_{d}=\left(\frac{\mu}{\varepsilon_{\mathrm{kin}}}\right)^{1 / 2} .
$$

This is motivated by the fact that the tracer particle dynamics are determined by the turbulent velocity field. The effects of the magnetic field are felt by the tracers indirectly via the distortions of the velocity field due to the Lorentz force. Note that the Kolmogorov length and time scales are denoted by $\ell_{d}$ and $\tau_{d}$ in this paper to prevent confusion with the magnetic diffusivity $\eta$.

In order to obtain quasistationary turbulence a large scale forcing is imposed by freezing all modes in the sphere $|\boldsymbol{k}| \leq k_{f}=2$ starting from a fully developed turbulent state. This leads to an energy transfer from frozen to freely evolving fluctuations via nonlinear interaction. In the resulting quasistationary state the total energy $E$ and energy dissipation rate $\varepsilon$ show only small fluctuations in time not exceeding $10 \%$. The Eulerian structure function scaling exponents agree in the Navier-Stokes case within errors with experiment (see, for example, Ref. 29) and in the MHD case with numerical $^{30}$ results. It has been checked that the large scale forcing does not introduce significant anisotropy by regarding direction-dependent Eulerian two-point statistics.

After a statistically stationary state is reached for both the velocity and the magnetic field, up to five million tracer particles are injected into the flow. In the case of simulations Run1 and Run2 the initial particle configuration consists of groups of tetrads $\left\{\left(\Delta_{0}, 0,0\right),\left(0, \Delta_{0}, 0\right),\left(0,0, \Delta_{0}\right)\right\}$ with an initial separation distance $\Delta_{0}$ that are placed on a randomly deformed cubic superlattice with a maximum perturbation of $25 \%$ per superlattice cell. This configuration represents a compromise between randomly distributed tetrads ${ }^{6}$ and a space filling and uniform tetrad distribution. ${ }^{7}$ In both Run1 and Run2 there are five groups of tetrads with different initial separation lengths $\Delta_{0}$ (see Table II) in order to probe different length scales of the turbulent velocity field. In the case of simulations of Run3 and Run4 the velocity field is seeded with randomly distributed tracers as these runs are mainly intended for the calculation of Lagrangian structure function scaling exponents.

The particle trajectories are integrated in time according to

$$
\frac{d \boldsymbol{X}(t)}{d t}=\boldsymbol{V}(t)=\boldsymbol{v}[\boldsymbol{X}(t), t]
$$

using a midpoint method. The instantaneous particle velocities $\boldsymbol{V}(t)$ are computed by tricubic polynomial interpolation of the velocity field $\boldsymbol{v}(\boldsymbol{x}, t)$, which has proven useful for high Reynolds number flows. ${ }^{31}$ The mean relative interpolation error has been estimated to be of the order of $0.1 \%$ by comparing tricubic interpolated values to Fourier-interpolated values on a turbulent velocity field. Particle positions $\boldsymbol{X}(t)$, their velocities $\boldsymbol{V}(t)$, and in the MHD case the magnetic field $\boldsymbol{B}(t)=\boldsymbol{b}[\boldsymbol{X}(t), t]$ at the instantaneous position of the particles have been sampled at a rate of $\approx \frac{1}{60} \tau_{d}$ for Run $1, \approx \frac{1}{80} \tau_{d}$ for Run2, $\approx \frac{1}{3} \tau_{d}$ for Run3, and $\approx \frac{1}{9} \tau_{d}$ for Run 4 .

TABLE II. Particle groups and respective initial pair separations $\Delta_{0}$ in simulations Run1 and Run2.

\begin{tabular}{lcccc}
\hline \hline & $\Delta_{0}$ (Run1) & $\Delta_{0}($ Run2) & $N_{\mathrm{p}}$ & Pairs \\
\hline Group 1 & $1.8 \ell_{d}$ & $2.1 \ell_{d}$ & $4.42 \times 10^{5}$ & $3.31 \times 10^{5}$ \\
Group 2 & $3.9 \ell_{d}$ & $4.6 \ell_{d}$ & $4.42 \times 10^{5}$ & $3.31 \times 10^{5}$ \\
Group 3 & $7.9 \ell_{d}$ & $9.2 \ell_{d}$ & $1.86 \times 10^{5}$ & $1.39 \times 10^{5}$ \\
Group 4 & $20 \ell_{d}$ & $23 \ell_{d}$ & $5.52 \times 10^{5}$ & $4.17 \times 10^{4}$ \\
Group 5 & $98 \ell_{d}$ & $115 \ell_{d}$ & $5.52 \times 10^{5}$ & $4.17 \times 10^{4}$ \\
\hline \hline
\end{tabular}




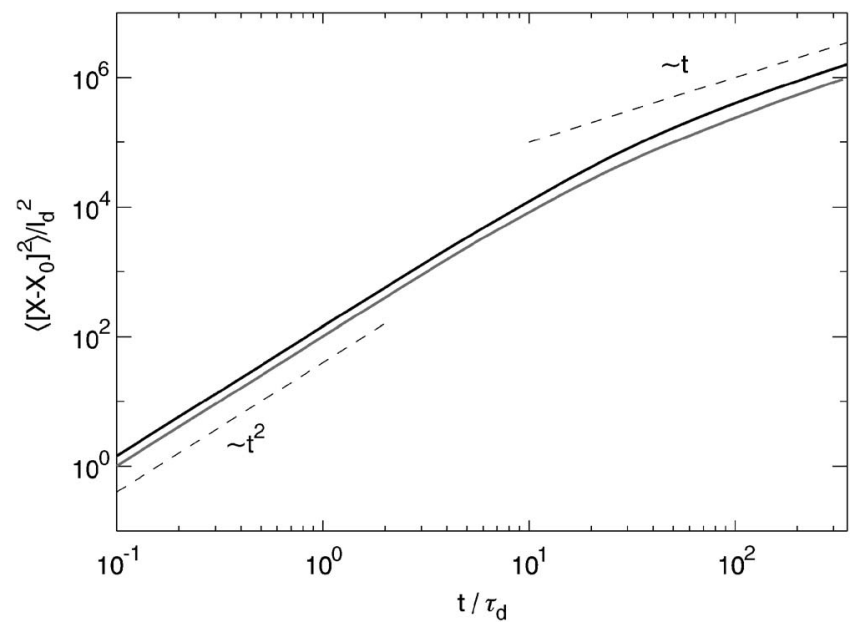

FIG. 1. Evolution of mean-square distance to initial position $\left\langle\left[\boldsymbol{X}(t)-\boldsymbol{X}_{0}\right]^{2}\right\rangle$ for turbulent single-particle diffusion in Navier-Stokes (black, Run1) and MHD (gray, Run2) turbulence. The dashed lines indicate ballistic scaling $\sim t^{2}$ and diffusive scaling $\sim t$. See also Ref. 33 .

\section{DIFFUSION STATISTICS}

The diffusion of single particles relative to a fixed source due to advection by a turbulent velocity field is characterized by the Lagrangian integral time scale

$$
T_{L}=\int_{0}^{\infty} d \tau \frac{\langle(\boldsymbol{V}(t) \boldsymbol{V}(t+\tau)\rangle}{\left\langle V^{2}\right\rangle} .
$$

$T_{L}$ is the autocorrelation time of the Lagrangian velocity calculated over all tracer trajectories. It was introduced by Taylor $^{32}$ to divide the single particle diffusion process in two time ranges with asymptotic behavior. The Lagrangian time scale $T_{L}$ in Run1 and Run 2 is of the same order of magnitude, $T_{L} \approx 16 \tau_{d}$ in the hydrodynamic and $T_{L} \approx 15 \tau_{d}$ in the magnetohydrodynamic case. The mean square displacement of the particles from their initial positions $\left\langle[\boldsymbol{X}(t)-\boldsymbol{X}(0)]^{2}\right\rangle$ is expected to grow quadratically with time for $t \ll T_{L}$ (ballistic scaling) and to show a diffusive dependence $\sim t$ for $t \gg T_{L}{ }^{32}$ In both simulations ballistic scaling is found (see Fig. 1) up to about $T_{L}$ and diffusive behavior for $t>50 \tau_{d}$. For $t>70 \tau_{d}$ finite-size effects as well as the influence of the large-scale forcing can be observed, as the particles have traveled then on average distances of half of the size of the periodic simulation volume. by

The normalized turbulent diffusion coefficient defined

$$
D_{\text {turb }}(t *)=\int_{0}^{t *} d \tau \frac{\langle\boldsymbol{V}(t) \boldsymbol{V}(t+\tau)\rangle}{\left\langle\boldsymbol{V}^{2}(t)\right\rangle},
$$

where $\langle\cdot\rangle$ denotes averaging over all tracer trajectories increases rapidly in the interval $0<t * \lesssim 50 \tau_{d}$ and then reaches its saturation value $T_{L}$.

With regard to turbulent single-particle diffusion no significant differences between the Navier-Stokes and the MHD system are observed. The slight offset in Fig. 1 can be explained by the lower level of kinetic energy in the magnetohydrodynamic case. This difference cannot be compensated entirely by the applied Kolmogorov normalization as the ki-

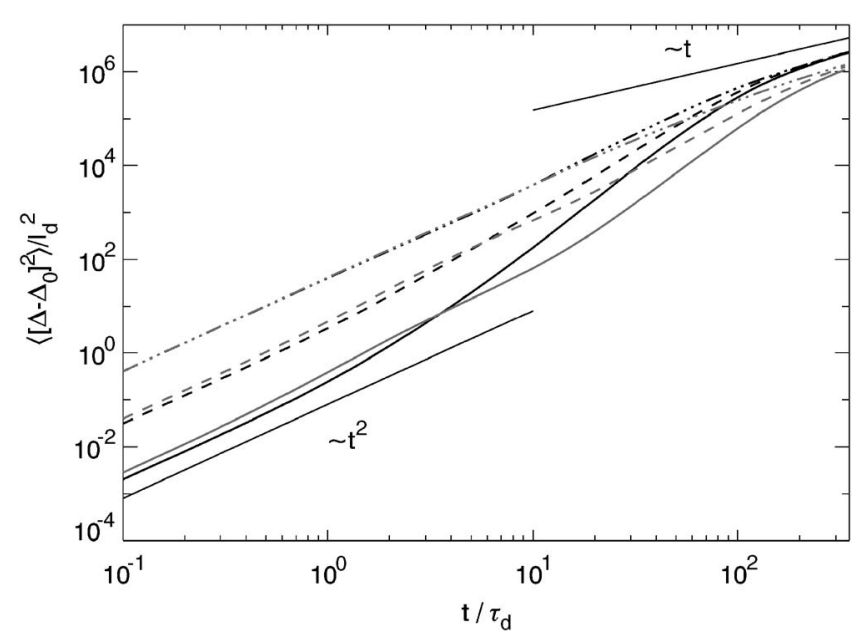

FIG. 2. Evolution of mean-square relative dispersion in Navier-Stokes (black) and MHD turbulence (gray). The behavior for three different initial pair-separations is shown; solid: group 1, dashed: group 3, dot-dashed: group 5 . The thin lines denote ballistic $\sim t^{2}$ and diffusive scaling $\sim t$. See also Ref. 33 .

netic energy dissipation rate $\varepsilon_{\text {kin }}$ is not linearly dependent on the kinetic energy. We do not find a slowing down of diffusion for MHD turbulence predicted analytically. ${ }^{34}$ However, in the cited work a delta-correlation in time of the velocity field has been assumed which neglects the dynamically important adaptation of the small scale velocity fluctuations to the magnetic field structure (see below).

\section{TWO-PARTICLE RELATIVE DISPERSION}

In contrast to turbulent diffusion of single tracers the relative motion of two particles (in the following denoted by the subscripts 1 and 2) is better suited for the investigation of the structural differences of the velocity field in the NavierStokes and the MHD system. In addition the dispersive properties of turbulence have great practical significance with regard to, e.g., the turbulent transport of passive contaminants and, in the MHD case, the stretching of magnetic field lines.

\section{A. Separation distance}

In homogeneous turbulence which is studied here the statistics of relative dispersion does not depend on the absolute position of the particles but only on their separation $\boldsymbol{\Delta}(t)=\boldsymbol{X}_{1}(t)-\boldsymbol{X}_{2}(t)$.

In the short time limit $t \ll T_{L}$ the mean squared relative dispersion $\left\langle\left[\boldsymbol{\Delta}(t)-\boldsymbol{\Delta}_{0}\right]^{2}\right\rangle$ is expected to grow quadratically with time since the relative velocity of the particles is approximately constant. In the large-time limit $t \gg T_{L}$ the particle-pair velocities become statistically independent; this results ultimately in a diffusive scaling of the mean square relative dispersion ${ }^{6,35}$

$$
\left\langle\left[\Delta(t)-\Delta_{0}\right]^{2}\right\rangle \sim \begin{cases}t^{2}, & \text { for } t \ll T_{L}, \\ t, & \text { for } t \gg T_{L} .\end{cases}
$$

We observe for both Run1 and Run2 $t^{2}$-scaling of the mean square relative dispersion shown in Fig. 2 for times up to $\tau_{d}$. For $t>160 \tau_{d}$ an approach to the diffusive limit is seen. 
Full decorrelation of the particle pair velocities is inhibited by the periodicity of the finite simulation volume. At intermediate times a period of acceleration is observed. The acceleration period is visibly delayed in the magnetohydrodynamic case; this feature will be investigated below in more detail.

For particles with initial separations in the inertial range there exist in the Navier-Stokes case predictions of Richardson $^{36}$ and Obukhov, ${ }^{37}$

$$
\left\langle\Delta^{2}(t)\right\rangle=g \varepsilon_{\mathrm{kin}} t^{3}, \quad t_{0} \ll t \ll T_{L},
$$

with the Richardson constant $g$, as well as of Batchelor ${ }^{35}$

$$
\left\langle\left[\Delta(t)-\Delta_{0}\right]^{2}\right\rangle=\frac{11}{3} C_{2}\left(\varepsilon_{\mathrm{kin}} \Delta_{0}\right)^{2 / 3} t^{2}, \quad t \ll t_{0},
$$

where $t_{0}=\left(\Delta_{0}^{2} / \varepsilon_{\mathrm{kin}}\right)^{1 / 3}$ can be interpreted as the time scale on which the eddies of size $\Delta_{0}$ evolve. The curves $\left\langle\left[\boldsymbol{\Delta}(t)-\boldsymbol{\Delta}_{0}\right]^{2}\right\rangle$ scaled by $\Delta_{0}^{2 / 3}$ of groups 4 and 5 almost collapse in both the Navier-Stokes and the MHD case on short time scales $t$ $\lesssim 5 \tau_{d}$. However, no exact Batchelor-scaling including all prefactors is observed which is probably due to the limited extent of the inertial range. Furthermore Richardson scaling entails $\Delta(t) \gg \Delta_{0}$ for $t \gg t_{0}$ with $\Delta(t)$ in the inertial range and an approach of the pair separation curves to one universal scaling law independent of $\Delta_{0}$. Hence, no Richardson scaling is found due to the moderate Reynolds numbers attained in our simulations. This is not unexpected in view of the fact that even in experiments at considerably higher Reynolds numbers no Richardson scaling has been observed. ${ }^{38}$ The Lyapunov exponent which characterizes the chaotic separation of infinitesimally close trajectories has not been computed as the calculation of the finite time Lyapunov exponent would have increased the computational cost considerably, exhausting the available resources. The alternative approach using the finite size Lyapunov exponent ${ }^{39}$ would involve the discussion of exit-time statistics which is beyond the scope of this paper.

For comparison with experiments and other numerical simulations an estimate of the value of the Richardson constant $g$ in the Navier-Stokes case has been made employing the method used in Refs. 6, 7, 12, and 13 by fitting a straight line to $\left\langle\Delta^{2}\right\rangle^{1 / 3}$ (not shown). The linear portion of the curve of group 2 which is closest to an approximate $t^{3}$ scaling gives $g \approx 0.50 \pm 0.05$. This result is of the same order of magnitude as found in previous studies. ${ }^{6,7,12,40}$ However, the value of $g$ must be treated with caution as no clear Richardson scaling is observed.

The probability density function (pdf) of the particle separation distance $P(\Delta)$ (see Fig. 3) first introduced by Richardson $^{36}$ as distance-neighbor function represents a comprehensive diagnostic of the pair-dispersion process. It exhibits a rapid change of shape in time also observed in Ref. 6.

The differences between the hydrodynamic and the MHD case are identified more easily in the time evolution of the skewness and flatness of the pdf of the separation distance defined as $S_{\Delta}=\left\langle\Delta^{3}\right\rangle /\left\langle\Delta^{2}\right\rangle^{3 / 2}$ (see Fig. 4) and $K_{\Delta}$ $=\left\langle\Delta^{4}\right\rangle /\left\langle\Delta^{2}\right\rangle^{2}$ (see Fig. 5), respectively.
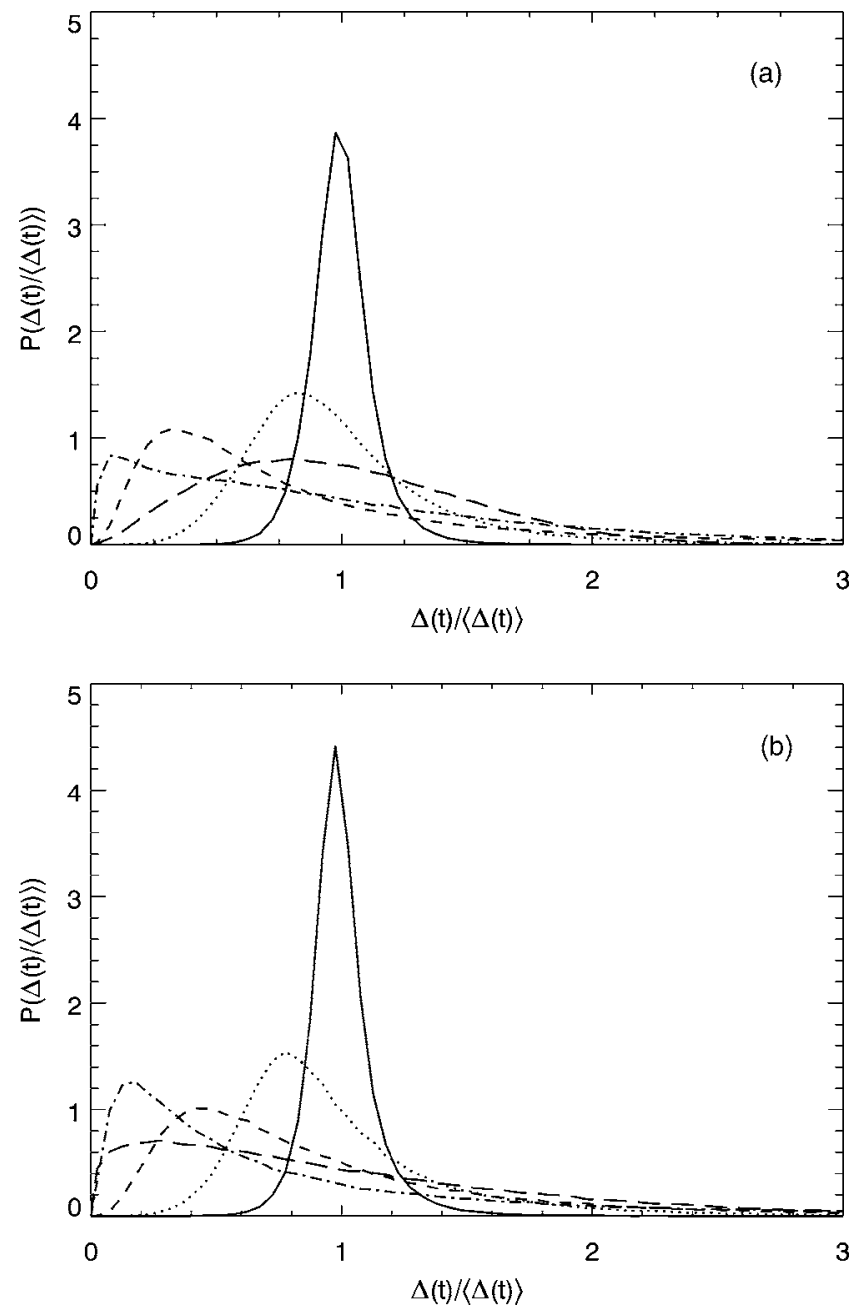

FIG. 3. The probability density function of the separation distance $\Delta$ in the Navier-Stokes (a) and MHD case (b) for the smallest initial separations $\Delta_{0}=1.8 \ell_{d}$ (Navier-Stokes case) and $\Delta_{0}=2.1 \ell_{d}$ (MHD case) at $t=0.5 \tau_{d}$ (solid line), $t=2 \tau_{d}$ (dotted line), $t=8 \tau_{d}$ (short dashed line), $32 \tau_{d}$ (dot-dashed line), and $128 \tau_{d}$ (long dashed line).

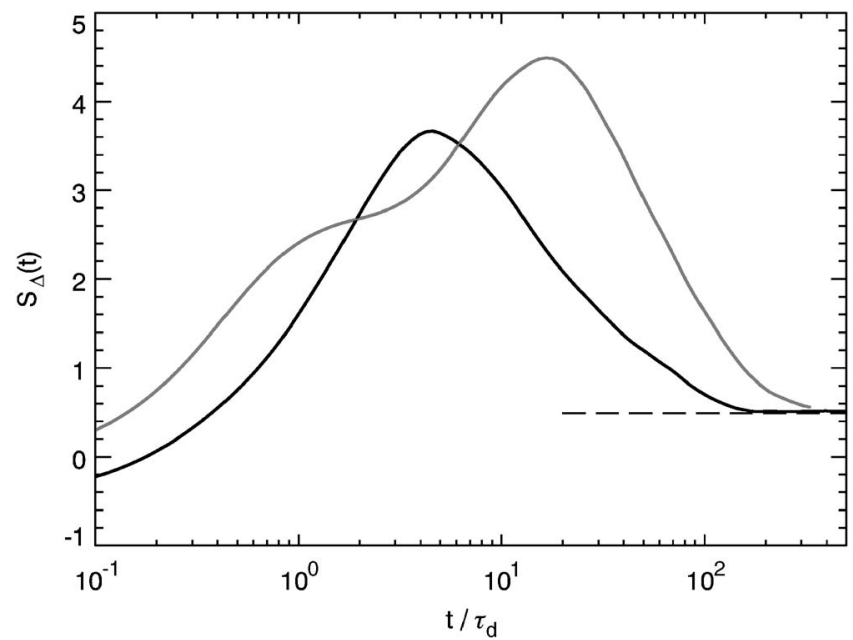

FIG. 4. The skewness of the pdf of the separation distance $\Delta$ for the smallest initial separations $\Delta_{0}=1.8 \ell_{d}$ (Navier-Stokes case, black) and $\Delta_{0}=2.1 \ell_{d}$ (MHD case, gray). The horizontal line is the appropriate chi-squared result of 0.49 . 


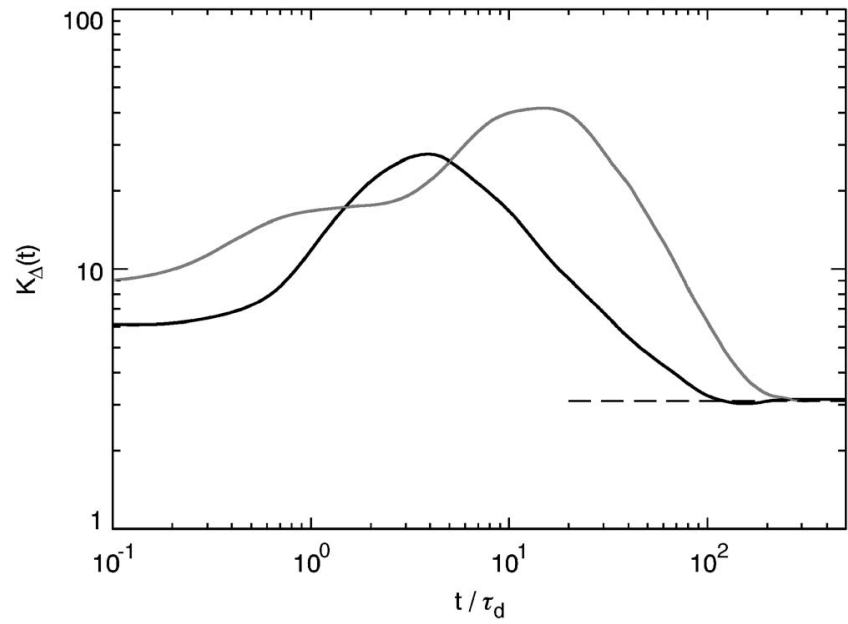

FIG. 5. The flatness of the pdf of the separation distance $\Delta$ for the smallest initial separations $\Delta_{0}=1.8 \ell_{d}$ (Navier-Stokes case, black) and $\Delta_{0}=2.1 \ell_{d}$ (MHD case, gray). The horizontal line is the appropriate chi-squared result of 3.1 .

In the short time limit negative (NS) and small (MHD) skewness and finite flatness are observed, since the relative velocity behaves on these time scales like a Eulerian velocity increment. ${ }^{6}$ In the long time limit the particle-pair displacements become statistically independent and the pdf of $\Delta^{2}$ is expected to approach a chi-squared distribution with three degrees of freedom (giving values for the skewness and flatness of the pdf of the separation distance of 0.49 and 3.1, respectively ${ }^{41}$ ).

At intermediate times the separation process is highly non-Gaussian in the Navier-Stokes case as also observed in Refs. 6 and 7 as well as in the MHD case. The maxima of flatness and skewness are in the MHD case even higher than in the Navier-Stokes case and they are reached at a later time.

For short times $t \lesssim \tau_{d}$ the level of intermittency, that is the aberration of the skewness and flatness from the corresponding chi-squared or Gaussian results, is visibly higher in the MHD case. Furthermore the period with high flatness and skewness values lasts longer in the MHD case.

The observed short-time behavior for times $t \lesssim \tau_{d}$ is explicable since the velocity fields are approximately constant in time during this period. The relative dispersion process is accordingly dominated by the spatial properties of the velocity fields as obtained by a Eulerian diagnostic. ${ }^{6}$ To clarify this point and for comparison with the Lagrangian observations the probability density function of the Eulerian longitudinal velocity increments $P\left(\delta v_{\ell}\right)$ with $\delta v_{\ell}=[\boldsymbol{v}(\boldsymbol{x}+\ell)$ $-\boldsymbol{v}(\boldsymbol{x})] \cdot \ell / \ell$ for an increment length of $\ell=d x$ is shown in Fig. 6. In the Navier-Stokes case a skewness of $-0.54 \pm 0.04$ is found which agrees within errors with experimental results $^{42}$ and a flatness of $6.6 \pm 0.3$. In the MHD case the probability density function is decidedly less skewed with a skewness of $-0.18 \pm 0.06$ and the tails are more pronounced (flatness 9.0 \pm 0.5 ) than in the Navier-Stokes case.

Skewness and flatness of the pdf of the separation distance show a rapid increase on short time scales because a particle pair with high initial relative velocity is more likely

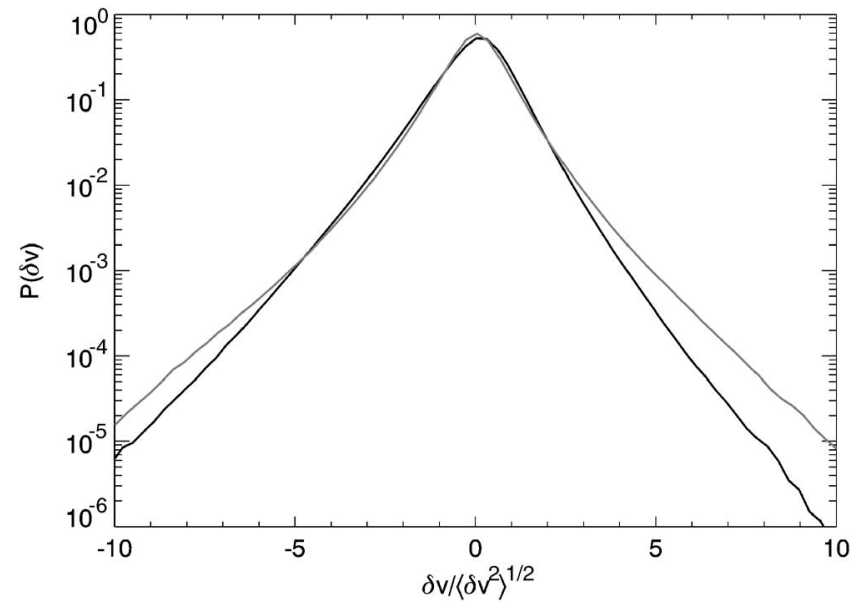

FIG. 6. Probability density functions of the Eulerian longitudinal increments of the velocity field $\delta v_{\ell}$ measured over a distance of $\ell=d x$ in the NavierStokes (black) and the MHD (gray) case.

to separate quickly. The separation process is apparently to some extend self-energizing as particle pairs with higher separation length are more likely to experience high velocity differences and will continue to separate even more rapidly. In the MHD case a higher number of particle pairs exist with extremely high relative velocities due to the pronounced tails of $P\left(\delta v_{\ell}\right)$. Therefore skewness and flatness attain higher values on short time scales $t<\tau_{d}$ in the MHD simulation compared to the NS values. In the subsequent time range from $\tau_{d} \lesssim t \lesssim 5 \tau_{d}$ flatness and skewness are approximately constant in the MHD case while in the Navier-Stokes case they rise to their maximum values.

In order to investigate the effect of the magnetic field in the MHD case we introduce the quantity $\overline{\boldsymbol{B}}=\left[\boldsymbol{b}\left(\boldsymbol{X}_{1}, t\right)\right.$ $\left.+\boldsymbol{b}\left(\boldsymbol{X}_{2}, t\right)\right] / 2$ as a rough proxy for the value of the local mean magnetic field at the particle pair position. In the MHD case the particle separation vector shows a tendency to align with the local magnetic field proxy in the above-mentioned time interval $\left(\tau_{d} \lesssim t \lesssim 5 \tau_{d}\right)$ due to the anisotropy of the relative velocity with respect to $\overline{\boldsymbol{B}}$ (see Sec. IV B). This can be observed in Fig. 7 where the probability density function of the angle $\kappa=\angle(\boldsymbol{\Delta}, \overline{\boldsymbol{B}})$ is shown.

In the case of an isotropic random orientation of $\boldsymbol{\Delta}$ and $\overline{\boldsymbol{B}}$ one would expect a sinusoidal distribution

$$
P(\kappa)=\frac{\pi}{360^{\circ}} \sin (\kappa) \text {. }
$$

As the direction of $\overline{\boldsymbol{B}}$ does not figure in the initial tetrad distribution $P(\kappa)$ starts from an approximately sinusoidal distribution at $t=0$. With increasing time the orientations parallel and antiparallel to $\overline{\boldsymbol{B}}$ become more and more favored. As a result of the alignment process the maximum values of flatness and skewness of the pdf of the separation distance are reached belatedly in the MHD case.

At $t \approx 8 \tau_{d} P(\kappa)$ has reached a quasistationary state, and no further increase of the alignment between $\boldsymbol{\Delta}$ and $\overline{\boldsymbol{B}}$ can be observed. The particle pairs continue to separate while the particle pair separation vector still shows a preferential align- 


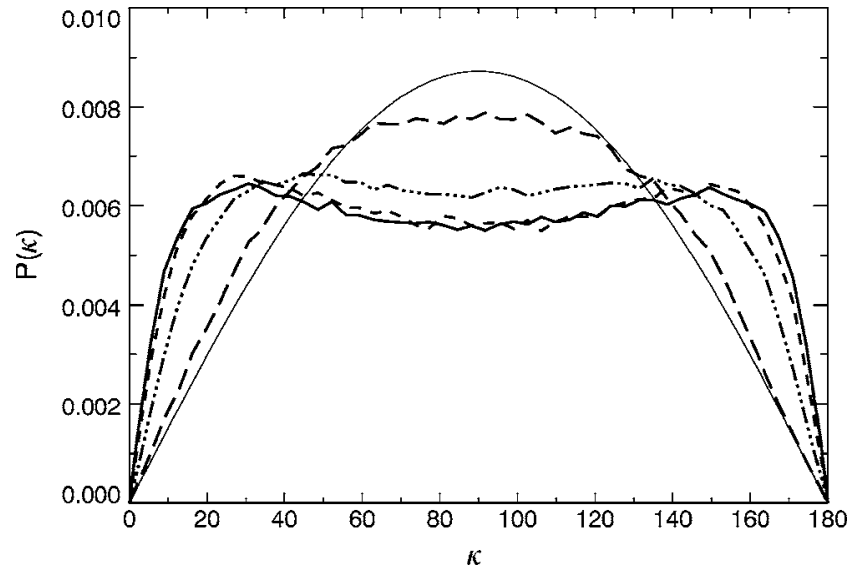

FIG. 7. Probability density function of the angle $\kappa$ between the separation vector $\Delta$ of particles in group 1 and the proxy of the local mean magnetic field $\overline{\boldsymbol{B}}$ at the times $\tau_{d}$ (long dashed), $4 \tau_{d}$ (dot-dashed), $8 \tau_{d}$ (short dashed), and $64 \tau_{d}$ (solid). The thin line denotes a sinusoidal distribution indicating isotropic random orientation of $\overline{\boldsymbol{B}}$ and $\boldsymbol{\Delta}$.

ment to the local mean magnetic field proxy. For long times the tracer movements become increasingly uncorrelated due to their growing separation distance. Eventually the differences between the NS and MHD system in the relative dispersion process become less pronounced as the skewness and flatness approach their theoretically predicted large time values.

\section{B. Relative velocity}

Studying the relative velocity of particle pairs yields further insight into the relative dispersion process. The relative velocity of a pair of tracer particles can be split into two parts

$$
\boldsymbol{U}(t)=\boldsymbol{V}_{1}(t)-\boldsymbol{V}_{2}(t)=U_{\|}(t) \boldsymbol{\Delta}(t) / \Delta(t)+\boldsymbol{U}_{\perp}(t),
$$

where $U_{\|}$is the component of the relative velocity parallel to the instantaneous particle separation vector $\boldsymbol{\Delta}$ and $\boldsymbol{U}_{\perp}$ is the perpendicular part. $U_{\|}$changes the length of the particle separation vector $\boldsymbol{\Delta}$, whereas $U_{\perp}$ alters the direction of $\boldsymbol{\Delta}$.

The mean parallel velocity (see Fig. 8) which is the

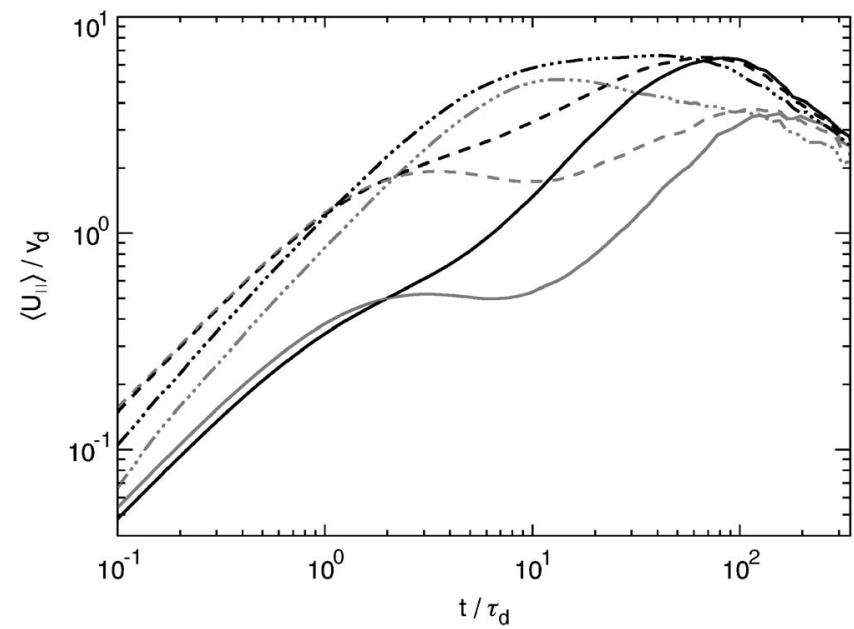

FIG. 8. Mean parallel velocity normalized by $v_{d}=\ell_{d} / \tau_{d}$ using the same symbols as in Fig. 2. Black lines: Navier-Stokes case, gray lines: MHD case.

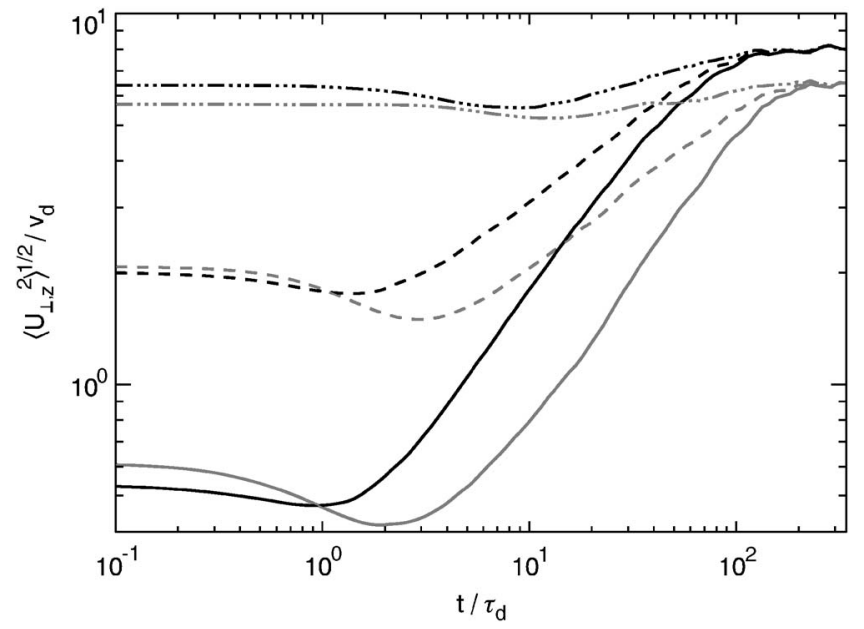

FIG. 9. Root-mean-square transverse relative velocity normalized by $v_{d}$ $=\ell_{d} / \tau_{d}$ (the $z$-component is shown, the same behavior is observed for the $x$ and $y$-components) using the same symbols as in Fig. 2. Black lines: NavierStokes case, gray lines: MHD case.

separation velocity by which the particle pairs are torn apart shows significant differences between the MHD case and the Navier-Stokes case. On short time scales a continuous increase of the separation velocity can be observed in Fig. 8. Then the growth of the separation velocity is slowed down as the particle pairs start to sense the temporal fluctuations of the velocity field, this time interval ranging roughly from $\tau_{d}$ to $10 \tau_{d}$ will be called the "slowing down period" in the following, although no actual deceleration takes place in the Navier-Stokes case. On larger time scales the separation velocity rises again more rapidly as the mean separation distance grows and the particles are dispersed by the more coherent large scale eddies. The separation velocity attains a maximum value at $t \approx 90 \tau_{d}$ (at $t \approx 150 \tau_{d}$ in the MHD case) and starts to decrease because of the periodicity of the simulation volume. The maximum of the separation velocity is reached later in the MHD case compared to the NS system because the mean separation distance reaches half of the length of the simulation volume at a later time. This is for two reasons: the lower kinetic energy in the MHD case as compared to the NS system and the slowing down period delaying particle spreading. The intermediate slowing down period is much more pronounced in the MHD case. While in the Navier-Stokes case the separation velocity still increases considerably in this time interval the separation velocity is approximately constant in the MHD case.

A similar behavior can be seen in the root-mean-square transverse velocity which is shown in Fig. 9 and which measures change of orientation of the separation vector $\Delta$. In the time interval of $0.5 \tau_{d} \lesssim t \lesssim 2 \tau_{d}$ the transverse velocity decreases in the MHD case. For later times $\left\langle U_{\perp}^{2}\right\rangle^{1 / 2}$ grows continuously to its saturation value. A slight decrease of $\left\langle U_{\perp}^{2}\right\rangle^{1 / 2}$ can be observed on short time scales $t \lesssim \tau_{d}$ in the NavierStokes case as well (see also Ref. 6) which can be attributed to the influence of temporal fluctuations of the velocity field. However, the decrease observed in the MHD case is much more pronounced.

The reason for the differences in the time evolution of 


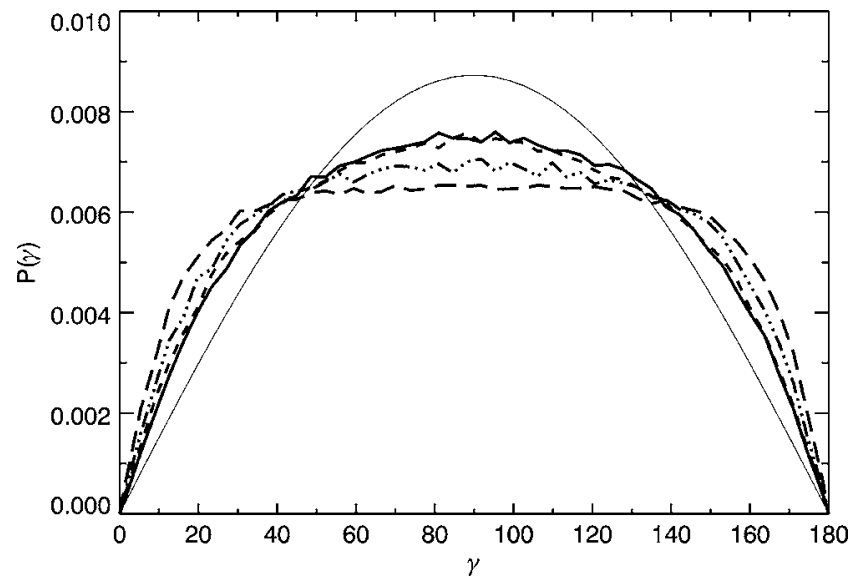

FIG. 10. Probability density function of the angle $\gamma$ between the relative velocity of particles in group 1 and the proxy of the local mean magnetic field $\overline{\boldsymbol{B}}$ for the times as in Fig. 7. The thin line denotes a sinusoidal distribution indicating isotropic random orientation of $\boldsymbol{V}$ and $\overline{\boldsymbol{B}}$. See also Ref. 33.

the relative velocity must be sought in the effect of the local mean magnetic field on the relative dispersion process. Although macroscopically the MHD system has no mean magnetic field, on small spatial scales the slowly evolving large scale magnetic field fluctuations act like a mean magnetic field. It is a well known fact that in magnetohydrodynamic turbulence turbulent eddies are anisotropic with respect to a mean magnetic field. ${ }^{43-48}$ As the fluid elements travel on average preferentially along the magnetic lines of force the relative dispersion is significantly reduced in the fieldperpendicular direction. Motions across field lines trigger quasi-oscillatory flow patterns which are supposed to drive the energy cascade ${ }^{44}$ but apparently do not lead to an effective separation of the particle pairs. This anisotropy causes the alignment of the particle separation vector $\Delta$ to the local mean magnetic field proxy $\overline{\boldsymbol{B}}$ as observed in Fig. 7 .

In order to support this conjecture the pdf of the angle $\gamma=\angle(\boldsymbol{U}, \overline{\boldsymbol{B}})$ for particle group 1 is shown at several points in time in Fig. 10 where $\overline{\boldsymbol{B}}$ is the local mean magnetic field proxy defined in the previous subsection. In the case of an isotropic random orientation of the velocity field with respect to $\overline{\boldsymbol{B}}$ a sinusoidal distribution [see Eq. (12)] would be expected. We observe, however, that on short time scales the distribution of the angle $P(\gamma)$ exhibits a clear deviation from this behavior favoring velocities aligned with $\overline{\boldsymbol{B}}$. A trend towards an isotropic distribution can be observed for large times which is due to the increase of the mean particle separation. The proxy $\overline{\boldsymbol{B}}$ is for large particle separations no longer a good approximation for the local mean magnetic field and becomes statistically independent of the separation velocity. Furthermore the effect of the magnetic field on large scale velocity fluctuations is not as pronounced as on fluctuations on smaller scales with significantly less kinetic energy.

As the particles start to separate preferentially along $\overline{\boldsymbol{B}}$ the relative dispersion in field perpendicular direction is reduced. This causes the observed plateau of the separation velocity shown in Fig. 8.

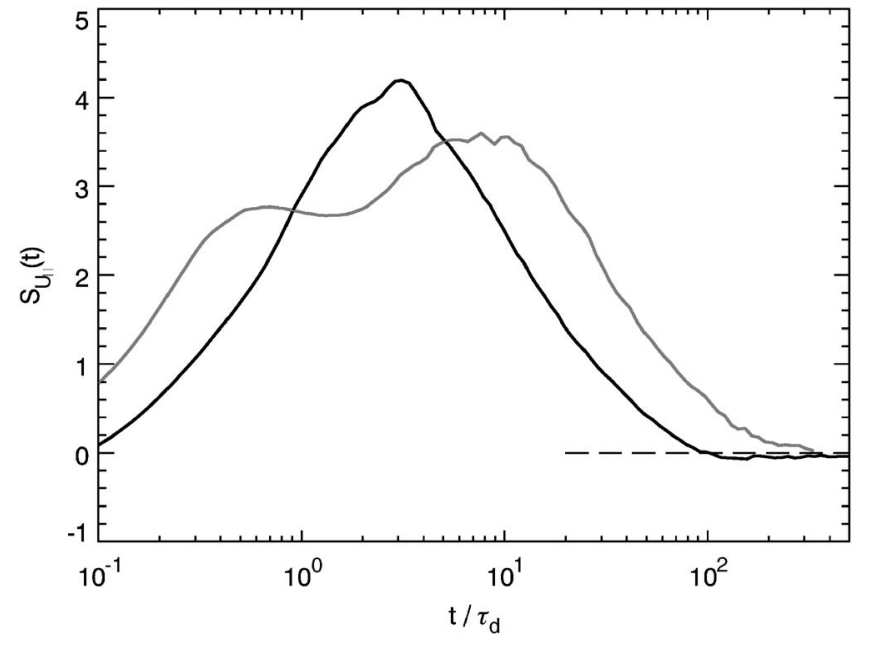

FIG. 11. The skewness of $P\left(U_{\|}\right)$for the Navier-Stokes (black line) and the MHD case (gray line) for group 1 . The dashed horizontal line indicates the Gaussian value of zero.

The $\overline{\boldsymbol{B}}$-perpendicular fluctuations of the velocity field contribute largely to the transverse relative velocity. As in the Navier-Stokes case the effect of temporal fluctuations probably leads to a decrease of $\left\langle U_{\perp}^{2}\right\rangle^{1 / 2}$ on short time scales. A further effect which might explain why the decrease of $\left\langle U_{\perp}^{2}\right\rangle^{1 / 2}$ is considerably higher in the MHD case is the observed alignment of the particle pair separation vector with $\overline{\boldsymbol{B}}$ which takes place in approximately the same time range. As the separation vectors align with the local mean magnetic field the $\overline{\boldsymbol{B}}$-parallel velocity fluctuations start to dominate the tracer dynamics and the influence of the field perpendicular velocity fluctuations decreases. As the mean separation distance is approximately constant in this time range (for particles of group 1 the mean separation distance has grown by $t=2 \tau_{d}$ only by a factor of 1.5$)$ this results in a decrease of $\left\langle U_{\perp}^{2}\right\rangle^{1 / 2}$.

A closer comparison of the time evolution of the relative velocities can be made by regarding skewness and flatness of

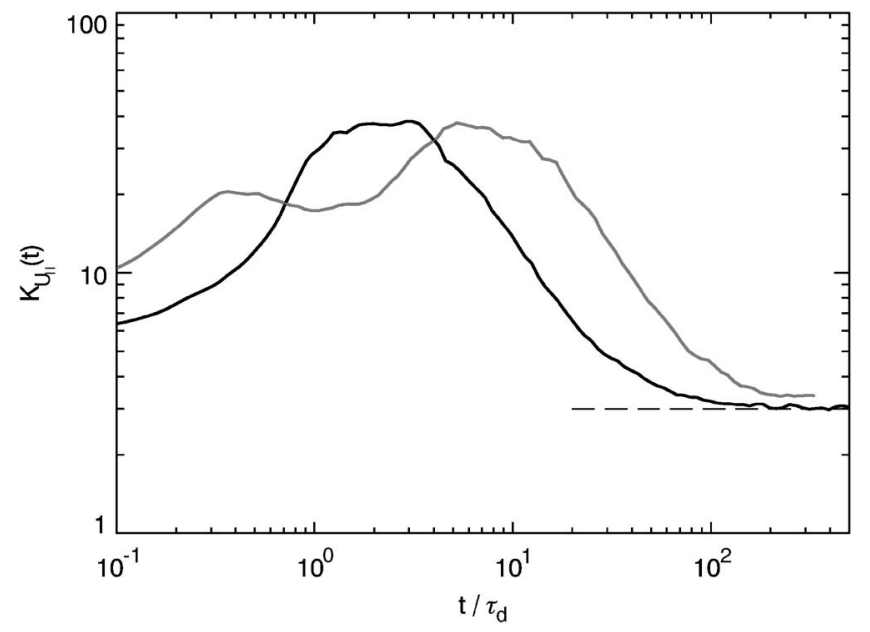

FIG. 12. The flatness of $P\left(U_{\|}\right)$for the Navier-Stokes (black line) and the MHD case (gray line) for group 1. The dashed horizontal line indicates the Gaussian value of 3 . 


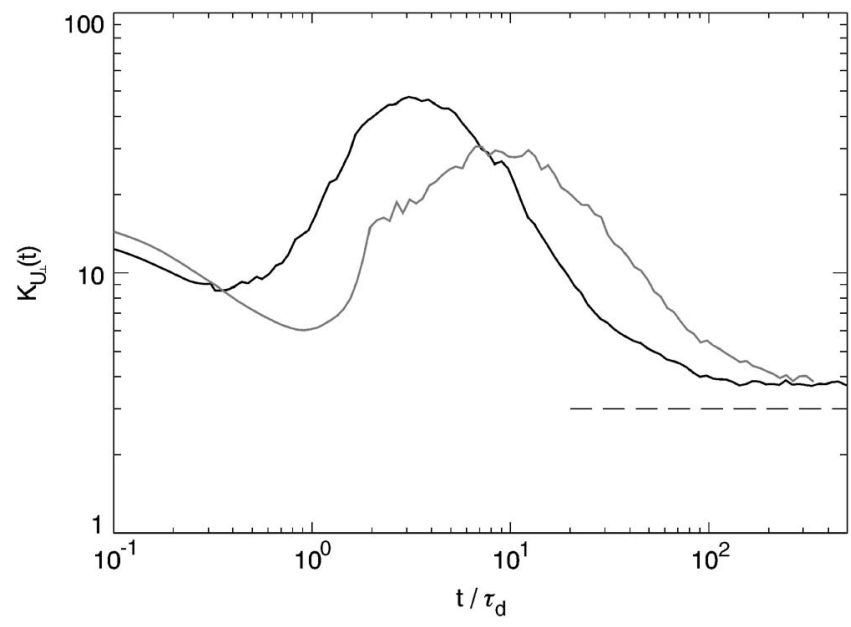

FIG. 13. The flatness of $P\left(U_{\perp, z}\right)$ for the Navier-Stokes (black line) and the MHD case (gray line) for group 1. The dashed horizontal line indicates the Gaussian value of 3 .

the pdfs of the parallel and transverse relative velocity components shown in Figs. 11-13. In the Navier-Stokes case we find basically the same time dependence that has been observed in previous simulations. ${ }^{6,7}$ The skewness of $P\left(U_{\|}\right)$ (see Fig. 11) rises from a negative value close to $-0.54 \mathrm{ob}-$ served in the probability density function of the Eulerian velocity increments (see Sec. IV A) to positive values. Both, skewness and flatness, reach their peak values which indicate the highest level of intermittency at $t \approx 4 \tau_{d}$ and approach Gaussian values in the long time limit. In the MHD case the period of maximal values in flatness and skewness starts earlier and lasts longer. Furthermore we discern two peaks, the first at about $t \approx 0.5 \tau_{d}$ and the second around $8 \tau_{d}$. For $t$ $\gtrsim 10 \tau_{d}$ we see again an approach to Gaussian values. Figure 13 shows the flatness for the pdf of the transverse component of the relative velocity which exhibits a pronounced decrease in the MHD case in the time range $0.1 \tau_{d} \lesssim t \lesssim 1 \tau_{d}$. In addition the maximum of the flatness is reached later than in the MHD case.

For a possible explanation of this behavior it is necessary to discern several time ranges. In the period of time which lasts approximately up to $0.5 \tau_{d}$ the motion of fluid particles is dominated by the spatial Eulerian characteristics of the turbulent velocity field which are discussed in the previous subsection. In MHD turbulence the pdf of the Eulerian velocity increments is not as negatively skewed as in the Navier-Stokes case and has a higher flatness. Therefore particle pairs with high separation velocities are more abundant in the MHD case in this time range. As a result skewness and flatness of $P\left(U_{\|}\right)$(see Figs. 11 and 12) as well as the flatness of $P\left(U_{\perp}\right)$ (see Fig. 13) are considerably higher (see Sec. IV A).

In the subsequent interval of time $0.5 \tau_{d} \lesssim t \lesssim 2 \tau_{d}$, the particle pairs begin to sense the turbulent fluctuations of the velocity field. As explained above the presence of the local mean magnetic field leads to a reduction of the dispersion in field-perpendicular direction and a slowing down of the relative dispersion process in the MHD case. Furthermore it must be taken into account that for a particle pair separation vector aligned to $\overline{\boldsymbol{B}}$ the component of the separation vector in the $\overline{\boldsymbol{B}}$-parallel direction is considerably larger than the perpendicular part. Therefore the influence of the $\overline{\boldsymbol{B}}$ perpendicular fluctuations of the velocity field on the particle pair decreases relative to the influence of the $\overline{\boldsymbol{B}}$-parallel component of the fluctuations. In addition it has been found that on small spatial scales turbulent fluctuations parallel to a mean magnetic field are smoother than the perpendicular fluctuations (see, e.g., Ref. 43). As a result the values of $\mathcal{K}\left(U_{\|}\right)$and $\mathcal{S}\left(U_{\|}\right)$stagnate and the flatness of the pdf of the transverse relative velocity decreases.

Once the separation process gains speed again the values of flatness and skewness increase as well and basically the same qualitative behavior as in the Navier-Stokes case can be observed. In the last time range $t>10 \tau_{d}$ an approach to Gaussian statistics can be observed as the particle velocities $\boldsymbol{V}_{1}$ and $\boldsymbol{V}_{2}$ become increasingly uncorrelated. A perfect decorrelation cannot be attained due to the finite extend of the simulation cube.

Particle pairs with higher initial separations of groups 2, 3 , and 4 show a similar behavior as members of group 1 . With rising initial separation the intermittency of the relative velocity decreases as also observed in Ref. 6. In the MHD case the alignment of the separation vector with the local mean magnetic field takes longer time which is why the above-mentioned time ranges are shifted to larger times. The particle pairs of group 5 with the highest initial separation show no intermittent behavior as their dynamics is dominated from the start by more coherent eddies at large scales.

\section{Error estimation}

Lagrangian statistics are known to be susceptible to extreme events in the fluctuating turbulent fields (see, e.g., Refs. 6 and 40). As a test for the reliability of the results within our statistical sample an estimate of the statistical error is calculated. The sample is divided into six subensembles of equal size and the maximum and minimum values of the separation distance and the skewness of the pdf of the relative velocity are calculated. In the Navier-Stokes case we find a maximum relative error of approximately $14 \%$ for the skewness of the pdf of the separation distance and approximately $19 \%$ for the skewness of the pdf of the longitudinal relative velocity. In the MHD case we find a maximum relative error of approximately $18 \%$ for both the separation skewness and the longitudinal relative velocity skewness. These errors are of the same order of magnitude as in previous numerical studies for the hydrodynamic case (see, for example, Refs. 6 and 7).

The periodicity of the simulation volume as well as the large scale forcing unavoidably affect the long time statistics of the Lagrangian tracers. These effects become visible for $t>70 \tau_{d}$.

\section{TRAJECTORIES AND STRUCTURE FUNCTIONS}

To get an impression of the different behavior of passive tracer particles in MHD and Navier-Stokes turbulence it is instructive to look at their trajectories directly. For the most 


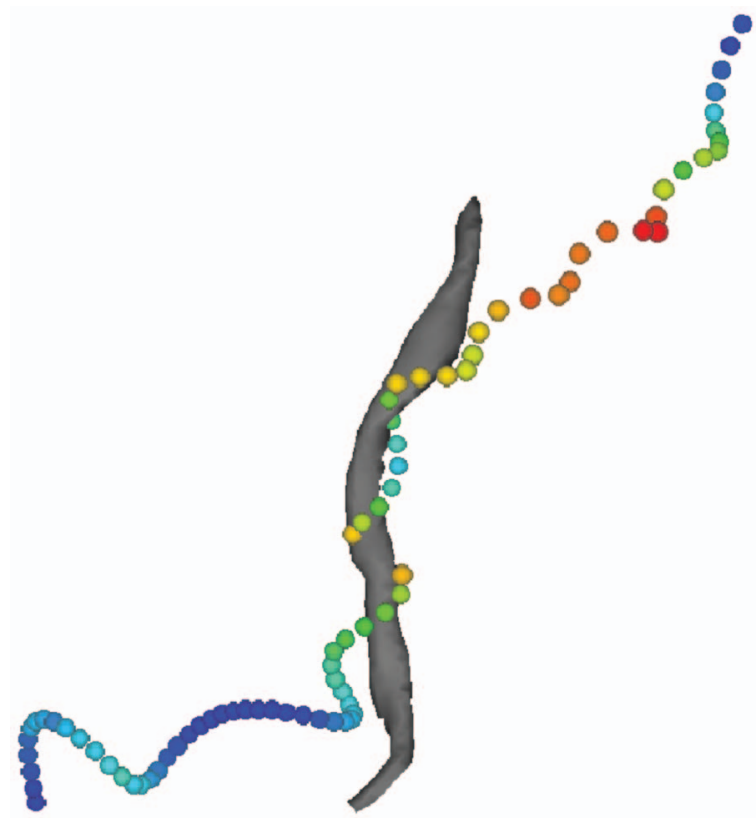

FIG. 14. (Color) Particle trajectory (blue corresponding to low acceleration, red to high acceleration) near a vortex filament in Navier-Stokes turbulence. The filament is shown at one instant in time.

part they are rather straight and uneventful. However, near highly dissipative structures, i.e., vortex filaments in the hydrodynamic case and vortex- and current-sheets in the MHD case, the tracer trajectories show characteristic shapes and the tracer particles experience high accelerations. In the hydrodynamic case the fluid particles tend to follow spiraling paths near vortex filaments (see Fig. 14) which is the socalled vortex trapping observed in numerical simulations. ${ }^{49}$ In the MHD case particles are often accelerated along vortex sheets and tend to show bends in their trajectories (see Fig. $15)$ in the vicinity of the vortex sheets. We observe two scenarios which cause high accelerations of tracer particles. In the first scenario tracers move along a sheet and hit another sheet nearly perpendicularly. The sudden change in the di-

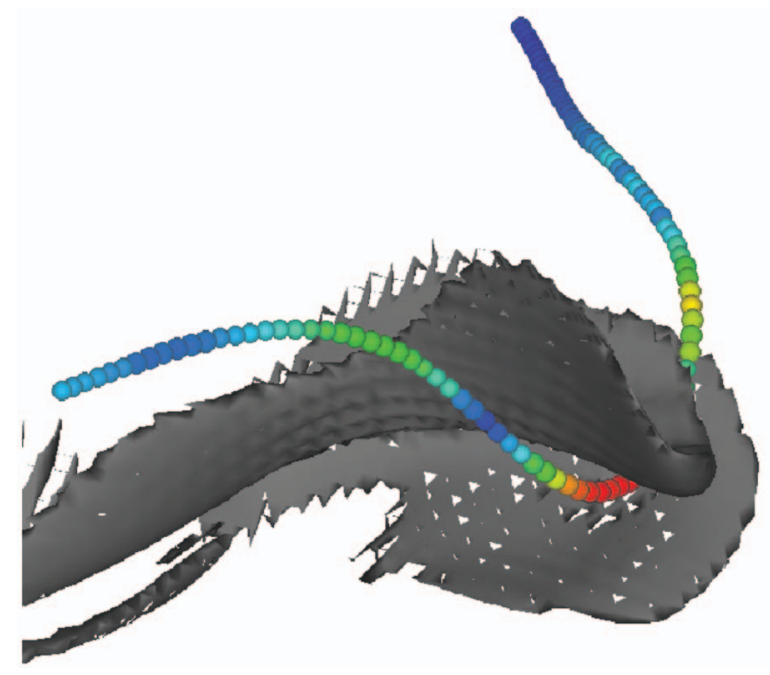

FIG. 15. (Color) Particle trajectory (blue corresponding to low acceleration, red to high acceleration) near vortex sheets in MHD turbulence.

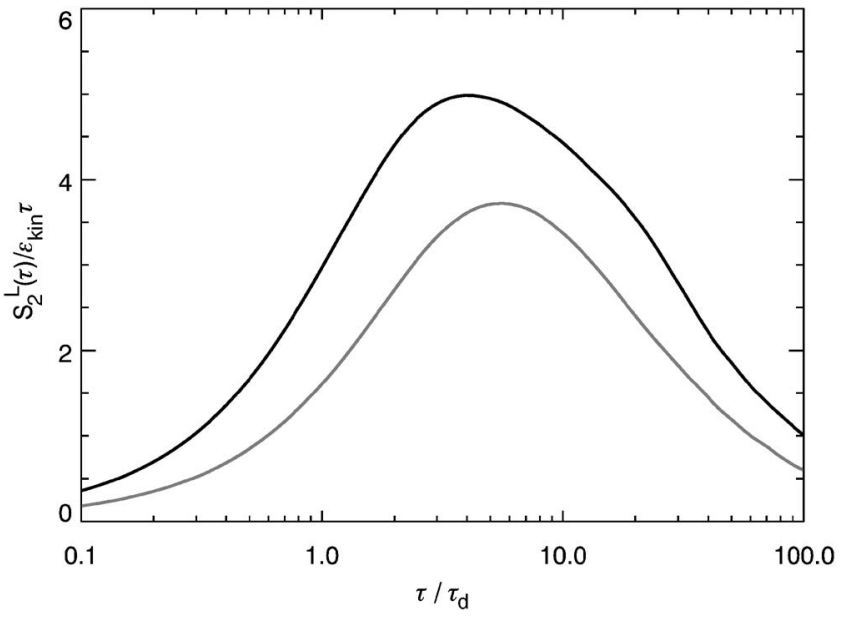

FIG. 16. Second order Lagrangian structure function normalized by kinetic energy dissipation rate $\varepsilon_{\text {kin }}$ and time increment $\tau$ for the hydrodynamic (black) and the MHD case (gray). Note that no scaling range can be observed.

rection of the trajectory implies high accelerations in a nearly point-like section of the path (see Ref. 8). In the second scenario tracers move along a bended vortex-sheet. Here also high accelerations occur in a small section of the trajectory compared to the size of the entire sheet. These two scenarios might also be related as can be seen in Fig. 15. As vortex and current sheets often appear in close vicinity to each other and have similar shapes a clear identification of the primary cause, i.e., current or vortex sheet, of the characteristic trajectories is not always possible.

By measuring the velocity increments along the particle trajectories one can calculate the Lagrangian velocity structure functions which are generally believed to display selfsimilar scaling in time

$$
S_{p}^{\mathrm{L}}(\tau)=\left\langle\left|V_{i}(t+\tau)-V_{i}(t)\right|^{p}\right\rangle \sim \tau^{\zeta^{\mathrm{L}}} .
$$

For the second order structure function one expects by dimensional analysis $\zeta_{p}^{L}=1,{ }^{50}$

$$
S_{2}^{L}(\tau)=C_{0} \varepsilon_{\text {kin }} \tau,
$$

where $C_{0}$ is the Lagrangian Kolmogorov constant.

This approach is motivated by the well-established importance of the Eulerian structure functions which involve spatial instead of temporal increments. Generally, structure functions supply a scale-dependent diagnostic of the turbulent fluctuations. This property renders them useful for measuring the intermittency of a turbulent field, i.e., the occurrence of extreme events on a fluctuating background. A general problem in the measurement of Lagrangian structure function scaling exponents from direct numerical simulations at the presently achievable resolutions ${ }^{51}$ is the absence of a Lagrangian inertial range (see Fig. 16). Recently it was shown $^{52}$ that a different Lagrangian increment derived from a Corrsin-type approximation yields a significantly larger scaling range than the standard increment. However, in this paper we stick to the conventional definition to allow comparison with other studies on Lagrangian two-point increments. 
TABLE III. Relative ESS-exponents calculated with respect to the second order structure function averaged over all three velocity components.

\begin{tabular}{lccccc}
\hline \hline & \multicolumn{2}{c}{ Navier-Stokes case } & & \multicolumn{2}{c}{ MHD case } \\
\cline { 2 - 3 } \cline { 5 - 6 }$p$ & Run1 & Run3 & & Run2 & Run4 \\
\hline 1 & $0.58 \pm 0.006$ & $0.57 \pm 0.006$ & & $0.527 \pm 0.004$ & $0.52 \pm 0.004$ \\
2 & 1 & 1 & & 1 & 1 \\
3 & $1.28 \pm 0.02$ & $1.31 \pm 0.02$ & & $1.41 \pm 0.02$ & $1.41 \pm 0.02$ \\
4 & $1.46 \pm 0.06$ & $1.50 \pm 0.06$ & & $1.76 \pm 0.04$ & $1.76 \pm 0.06$ \\
5 & $1.58 \pm 0.12$ & $1.70 \pm 0.10$ & & $2.05 \pm 0.08$ & $2.06 \pm 0.12$ \\
6 & $1.67 \pm 0.19$ & $1.82 \pm 0.14$ & & $2.30 \pm 0.13$ & $2.30 \pm 0.21$ \\
\hline \hline
\end{tabular}

One has to add that even at high Reynolds numbers an inertial scaling of the Lagrangian structure functions does not necessarily occur as the velocity increments calculated for a single particle may be affected by the nonuniversal large scale structure of the turbulent fields.

For want of a clear scaling range we selected the region where the second order structure function scales approximately linear in $t$ to measure the relative scaling exponents $\zeta_{p}^{L} / \zeta_{2}^{L}$ using extended self-similarity. ${ }^{53}$

Due to the considerable duration of the simulation runs we were able to calculate the Lagrangian structure functions at several different points of time $t$ separated by several $T_{L}$ in order to ensure statistical independence of the different samples. The resulting relative ESS-exponents for two independent simulations each in the Navier-Stokes and MHDcase are shown in Table III. The results in the Navier-Stokes case agree within errors with recent experimental findings. 5,54

In Ref. 49 the scaling range has been chosen for time increments $\tau=20 \tau_{d}-50 \tau_{d}$ which results in different values for the structure function scaling exponents. However, it has to be noted that for larger time increments $\tau$ the influence of the large scale forcing and the periodic boundary conditions can affect the structure functions. Therefore we have chosen to follow the approach of $\mathrm{Xu}$ et al., in Ref. 54 in the selection of our scaling range. Note that the Lagrangian structure functions show a more intermittent behavior in the Navier-

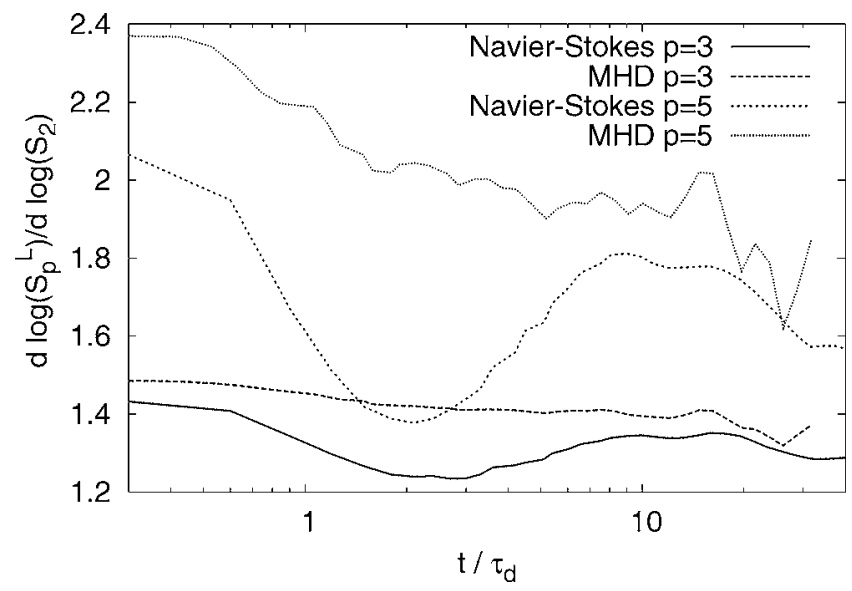

FIG. 17. Logarithmic derivative of relative structure functions for Run3 and Run4.

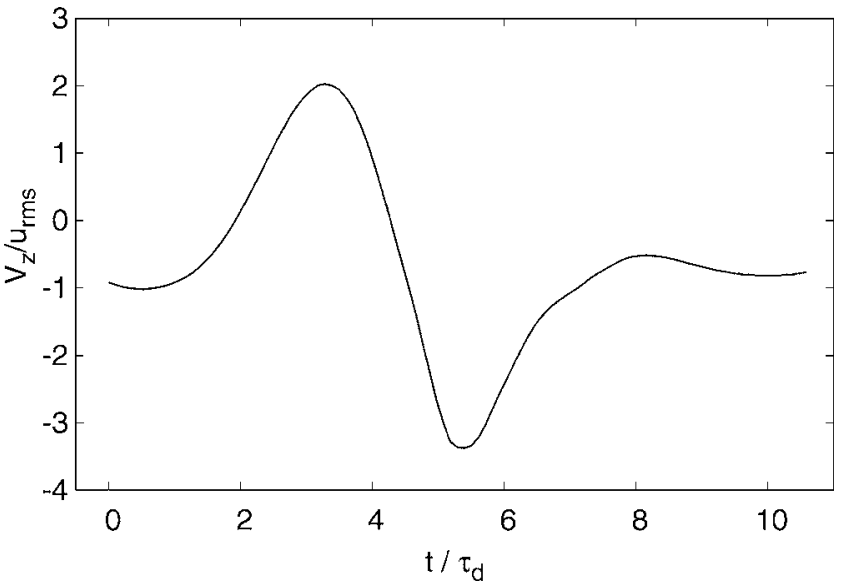

FIG. 18. Time dependence of velocity ( $z$-direction) for particle trajectory shown in Fig. 15 (MHD case).

Stokes case than in the MHD case for larger time increments as well. This can be seen from Fig. 17. Here, the logarithmic derivative of relative Lagrangian structures functions are given for two different orders in Navier-Stokes and MHD turbulence. Whatever range one chooses for evaluating the scaling exponents the MHD values are larger than the Navier-Stokes ones. A second qualitative conclusion can be drawn from Fig. 17. The Navier-Stokes functions display a pronounced knee at a few Kolmogorov times. This can be attributed to trapping events of tracers in coherent vortex filaments (see Ref. 55). The corresponding MHD functions display no knee. This is in line with the observation that in MHD turbulence no trapping events occur.

The lower degree of intermittency indicated by the Lagrangian structure functions in the MHD case is surprising as the MHD Eulerian structure functions show a higher degree of intermittency than in the Navier-Stokes case. ${ }^{30}$ This apparent contradiction can be explained by regarding the particle trajectories. In hydrodynamic turbulence particle trapping events have been found to enhance the intermittency of the Lagrangian structure functions for times up to $10 \tau_{d}{ }^{49}$ In our MHD-runs we could not observe particle trapping events. The corresponding bended trajectories near vortex sheets do not have a similar intermittency enhancing effect. Although the particles experience large accelerations near vortex sheets, a deflected particle shows basically a single large velocity increment as the direction of its velocity is turned (see Fig. 18) whereas a trapped particle gyrating rapidly experiences many large velocity increments because its velocity changes its direction perpetually (see Fig. 19).

From the second order structure function normalized by $\varepsilon_{\text {kin }} \tau$ one can estimate the value of the Lagrangian Kolmogorov constant $C_{0}$ in Eq. (15). The Lagrangian Kolmogorov constant plays an important role in the stochastic modelling of Lagrangian statistics (see, for example, Ref. 10). In the Navier-Stokes case we find a value of $C_{0}=4.9 \pm 0.2$ (Run1) which is of the same order of magnitude as previously measured experimental and numerical values (see, for example, Refs. 6 and 56). In the MHD case the value of $C_{0}$ is somewhat smaller, $C_{0}=3.9 \pm 0.2$ (Run2). The respective values for Run3 and Run4 are consistent with these findings. As this 


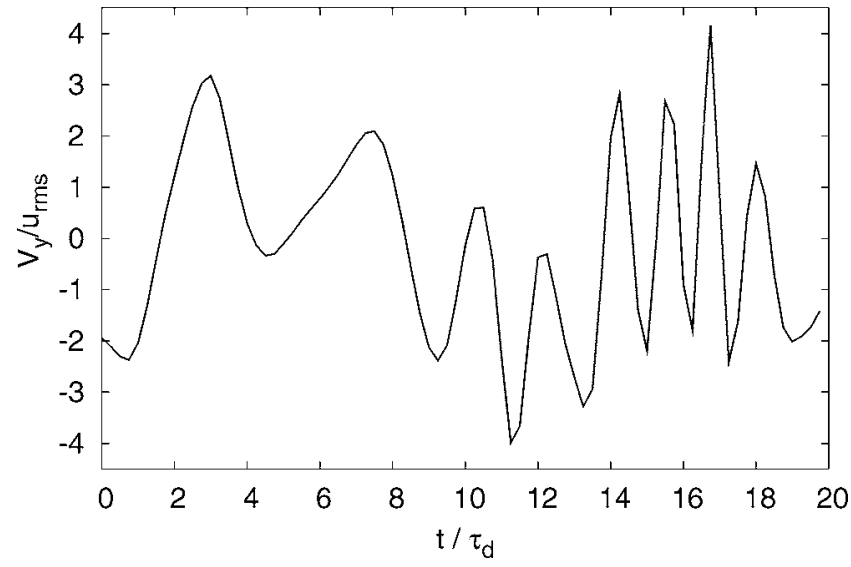

FIG. 19. Time dependence of velocity ( $y$-direction) for particle trajectory shown in Fig. 14 (Navier-Stokes case).

parameter is of importance in some statistical models of relative dispersion ${ }^{10}$ the lower value in the MHD case might be connected to the slower relative dispersion discussed in Sec. IV.

\section{CONCLUSIONS}

This paper presents a detailed comparison of the Lagrangian statistics of incompressible and macroscopically isotropic Navier-Stokes and MHD turbulence. To this end data generated by several high-resolution direct numerical simulations is analyzed.

Simple diffusion does not exhibit significant differences between the MHD and the Navier-Stokes system, whereas for the relative dispersion of particle pairs many qualitative differences are observed. Both the particle pair distance and the relative velocity show a different time evolution in the MHD case. Due to the constricting effect of the local mean magnetic field the relative dispersion is slowed down. The anisotropy of the small scale turbulent eddies with respect to the local mean magnetic field leads to an alignment of the particle-pair separation vector with the local mean magnetic field. This shows that even in cases where no global mean magnetic field exists the large scale fluctuations of the magnetic field lead to an anisotropy in the relative dispersion process.

The tracer particle trajectories show near structures of high dissipation characteristic shapes. In the Navier-Stokes case near vortex filaments spiraling trajectories are found, whereas in the MHD case deflected trajectories are observed near vortex sheets. This difference in the characteristic shapes of the trajectories might be responsible for the lower level of intermittency of the Lagrangian structure functions in the MHD case compared to the Navier-Stokes case.

\section{ACKNOWLEDGMENTS}

A.B. and W.C.M. gratefully acknowledge support by Lorenz Kramer and Walter Zimmermann at the University of Bayreuth. Computations were performed on the Altix 3700 system at the Leibniz-Rechenzentrum, Munich; the JUMP multiprocessor at Forschungszentrum Jülich through Project No. HB022; and on a Linux-Opteron Cluster supported by the HBFG-108-291.

The work of H.H. and R.G. benefitted from support through SFB 591 of the Deutsche Forschungsgesellschaft.

${ }^{1}$ A. La Porta, G. A. Voth, F. Moisy, and E. Bodenschatz, Phys. Fluids 12, 1485 (2000).

${ }^{2}$ N. Mordant, P. Metz, O. Michel, and J.-F. Pinton, Phys. Rev. Lett. 87, 214501 (2001).

${ }^{3}$ A. La Porta, G. A. Voth, A. M. Crawford, J. Alexander, and E. Bodenschatz, Nature (London) 409, 1017 (2001).

${ }^{4}$ G. A. Voth, A. La Porta, A. M. Crawford, E. Bodenschatz, C. Ward, and J. Alexander, Rev. Sci. Instrum. 72, 4348 (2001).

${ }^{5}$ N. Mordant, E. Lévêque, and J.-F. Pinton, New J. Phys. 6, 116 (2004).

${ }^{6}$ P. K. Yeung and M. S. Borgas, J. Fluid Mech. 503, 93 (2004).

${ }^{7}$ L. Biferale, G. Boffetta, A. Celani, B. J. Devenish, A. Lanotte, and F. Toschi, Phys. Fluids 17, 115101 (2005).

${ }^{8}$ H. Homann, R. Grauer, A. Busse, and W.-C. Müller, J. Plasma Phys. 73, 821 (2007)

${ }^{9}$ P. K. Yeung, Annu. Rev. Fluid Mech. 34, 115 (2002).

${ }^{10}$ B. Sawford, Annu. Rev. Fluid Mech. 33, 289 (2001).

${ }^{11}$ G. A. Voth, K. Satyanarayan, and E. Bodenschatz, Phys. Fluids 10, 2268 (1998).

${ }^{12}$ S. Ott and J. Mann, J. Fluid Mech. 422, 207 (2001).

${ }^{13}$ T. Ishihara and Y. Kaneda, Phys. Fluids 14, L69 (2002).

${ }^{14}$ P. Pommois, G. Zimbardo, and P. Veltri, Nonlinear Processes Geophys. 8, 151 (2001)

${ }^{15}$ J. R. Jokipii and E. N. Parker, Astrophys. J. 155, 777 (1969).

${ }^{16}$ J. Giacalone and J. R. Jokipii, Astrophys. J. 520, 204 (1999).

${ }^{17}$ J. Maron, B. D. G. Chandran, and E. Blackman, Phys. Rev. Lett. 92, 045001 (2004).

${ }^{18}$ W. H. Matthaeus, P. C. Gray, D. H. Pontius, Jr., and J. W. Bieber, Phys. Rev. Lett. 75, 2136 (1995).

${ }^{19}$ F. Cattaneo, Astrophys. J. 434, 200 (1994)

${ }^{20}$ A. B. Rechester and M. N. Rosenbluth, Phys. Rev. Lett. 40, 38 (1978).

${ }^{21}$ J. A. Krommes, C. Oberman, and R. G. Kleva, J. Plasma Phys. 30, 11 (1983).

${ }^{22}$ M. Isichenko, Plasma Phys. Controlled Fusion 33, 809 (1991).

${ }^{23}$ A. Pumir, B. I. Shraiman, and M. Chertkov, Phys. Rev. Lett. 85, 5324 (2000).

${ }^{24}$ G. Falkovich, K. Gawędzki, and M. Vergassola, Rev. Mod. Phys. 73, 913 (2001).

${ }^{25}$ D. Biskamp, Magnetohydrodynamic Turbulence (Cambridge University Press, Cambridge, 2003), pp. 10-32.

${ }^{26}$ A. Vincent and M. Meneguzzi, J. Fluid Mech. 225, 1 (1991).

${ }^{27}$ T. Y. Hou and R. Li, J. Comput. Phys. 226, 379 (2007).

${ }^{28}$ P. K. Yeung and S. B. Pope, J. Fluid Mech. 207, 531 (1989).

${ }^{29}$ A. Arneodo, C. Baudet, F. Belin, R. Benzi, B. Castaing, B. Chabaud, R. Chavarria, S.Ciliberto, R. Camussi, F. Chilla, B. Dubrulle, Y. Gagne, B. Hebral, J. Herweijer, M. Marchand, J. Maurer, J. F. Muzy, A. Naert, A. Noullez, J. Peinke, F. Roux, P. Tabeling, W. vandeWater, and H. Williame, Europhys. Lett. 34, 411 (1996).

${ }^{30}$ W.-C. Müller and D. Biskamp, Phys. Rev. Lett. 84, 475 (2000).

${ }^{31}$ H. Homann, J. Dreher, and R. Grauer, Comput. Phys. Commun. 177, 560 (2007).

${ }^{32}$ G. I. Taylor, Proc. London Math. Soc. 20, 196 (1922).

${ }^{33}$ W.-C. Müller and A. Busse, Europhys. Lett. 78, 14003 (2007).

${ }^{34}$ E. Kim, Phys. Plasmas 7, 1746 (2000).

${ }^{35}$ G. K. Batchelor, Q. J. R. Meteorol. Soc. 76, 133 (1950).

${ }^{36}$ L. F. Richardson, Proc. R. Soc. London, Ser. A 110, 709 (1926)

${ }^{37}$ A. M. Obukhov, Izv. Akad. Nauk SSSR, Ser. Geogr. Geofiz. 5, 453 (1941).

${ }^{38}$ M. Bourgoin, N. Ouellette, H. Xu, J. Berg, and E. Bodenschatz, Science 311, 835 (2006).

${ }^{39}$ E. Aurell, G. Boffetta, A. Crisanti, G. Paladin, and A. Vulpiani, J. Phys. A 30, 1 (1997)

${ }^{40}$ G. Boffetta and I. M. Sokolov, Phys. Rev. Lett. 88, 094501 (2002).

${ }^{41}$ P. K. Yeung, Phys. Fluids 6, 3416 (1994).

${ }^{42}$ K. R. Sreenivasan and R. A. Antonia, Annu. Rev. Fluid Mech. 29, 435 (1997).

${ }^{43}$ W.-C. Müller, D. Biskamp, and R. Grappin, Phys. Rev. E 67, 066302 (2003). 
${ }^{44}$ P. Goldreich and S. Sridhar, Astrophys. J. 438, 763 (1995).

${ }^{45}$ J. Cho and E. T. Vishniac, Astrophys. J. 539, 273 (2000).

${ }^{46}$ J. Maron and P. Goldreich, Astrophys. J. 554, 1175 (2001).

${ }^{47}$ J. Cho, A. Lazarian, and E. T. Vishniac, Astrophys. J. 564, 291 (2002).

${ }^{48}$ J. V. Shebalin, W. H. Matthaeus, and D. Montgomery, J. Plasma Phys. 29, 525 (1983).

${ }^{49}$ L. Biferale, G. Boffetta, A. Celani, A. Lanotte, and F. Toschi, Phys. Fluids 17, 021701 (2005).

${ }^{50}$ L. D. Landau and E. M. Lifshitz, Fluid Mechanics (Pergamon, London, 1959), pp. 120-123.

${ }^{51}$ P. K. Yeung, S. B. Pope, and B. L. Sawford, J. Turb. 7, 1 (2007).
${ }^{52}$ R. Friedrich, O. Kamps, H. Homann, and R. Grauer, "Relations between Eulerian and Lagrangian velocity increment statistics in fully developed turbulence," Phys. Lett. A (submitted).

${ }^{53}$ R. Benzi, S. Ciliberto, R. Tripiccione, C. Baudet, F. Massaioli, and S. Succi, Phys. Rev. E 48, R29 (1993).

${ }^{54} \mathrm{H}$. Xu, M. Bourgoin, N. Ouellette, and E. Bodenschatz, Phys. Rev. Lett. 96, 024503 (2006).

${ }^{55}$ J. Bec, L. Biferale, M. Cencini, F. Lanotte, and A. Toschi, Phys. Fluids 18, 081702 (2006).

${ }^{56}$ R.-C. Lien and E. A. D'Asoro, Phys. Fluids 14, 4456 (2002). 